Chapter 5

\title{
Recent Developments of Single Photon Emission Computed Tomography for the Diagnosis of Brain Tumors
}

\author{
Yasushi Shibata \\ Additional information is available at the end of the chapter \\ http://dx.doi.org/10.5772/52352
}

\section{Introduction}

1.1. Diagnostic ability of ${ }^{201}$ thallium SPECT and ${ }^{99}$ technetium methoxyisobutylisonitrile SPECT for patients with initial glioma

Single photon emission computed tomography (SPECT) is a valuable diagnostic modality for the evaluation of brain tumor malignancy and activity. Thallium-201 (Tl) SPECT and Technetium-99m methoxyisobutylisonitrile (Tc-MIBI) SPECT were clinically used to evaluate brain tumor malignancy and activity. In addition, both early and delayed images were taken depending on the preference of each institute. The results of SPECT examinations always include some false positive or false negative findings. The diagnostic value of brain tumor SPECT has been evaluated using sensitivities and specificities with arbitrary cut off values [1-8]. These arbitrary cut off values depend on the measurement methods used by each institute, as a result, studies using these arbitrary cut off values are not useful for evaluating tumor malignancy at other institutes.

A receiver operating characteristic (ROC) analysis is useful for evaluating the diagnostic ability of different examinations that include some errors $[9,10]$. In order to investigate the ability of each SPECT modality to evaluate tumor malignancy, each SPECT modality was directly compared for the patients with an initial glioma using an ROC analysis. The hypothesis is that the one SPECT modality is superior to any other modalities for grading glioma.

\subsection{Methods}

The study population included 59 patients with glioma who were admitted to Tsukuba University hospital between 1999 and 2005 and who underwent SPECT imaging. None 
of the patients had received any previous radiation therapy to the brain and recurrent cases were excluded. $\mathrm{Tl}$ and Tc-MIBI SPECT images were taken before surgery, radiation or chemotherapy in most patients. The SPECT images were taken after surgery but before radiation or chemotherapy in one patient. All pathological diagnoses were confirmed after the surgical removal of the tumor. The benign group included low grade astrocytomas $(n=23)$ and a central neurocytoma $(n=1)$ while the malignant group included anaplastic astrocytomas $(n=10)$ and glioblastomas $(n=25)$. The benign group included 13 men and 11 women, ranging from 3 to 59 years of age and the median age was 32 years old. The malignant group included 17 men and 18 women, ranging from 7 to 79 years of age and the median age was 55 years old.

Tl SPECT and Tc-MIBI SPECT images were obtained $15 \mathrm{~min}$ (early) and $3 \mathrm{hr}$ (delayed) after the intravenous injection of $74 \mathrm{MBq}$ of $\mathrm{Tl}$ chloride or $740 \mathrm{MBq}$ of Tc-MIBI using a multi-detector SPECT machine (E.CAM, Siemens Medical, Malvern, PA) and a high resolution collimator (LEHR, Siemens Medical, Malvern, PA). The Butterworth pre-correction filter and the Chang method were used for pre and post attenuation corrections. The Ramp filter was used for reconstruction. The image matrixes for Tl and MIBI SPECT were 64x64 and 128x128. The pixel sizes for Tl and MIBI SPECT were $6.61 \mathrm{~mm}$ and $3.31 \mathrm{~mm}$. The slice thickness both of $\mathrm{Tl}$ and MIBI SPECT was $6.61 \mathrm{~mm}$.

The Regions of Interest (ROI) were set on tumor and contra-lateral normal white matter in reconstructed SPECT images. The ROIs were customized for each patient. In the cases with a hot tracer uptake into the tumor, the ROIs were placed at homogeneously high uptake areas. In cases with no tracer uptake, the ROIs were placed at suspected tumor areas using the MRI findings as references. All ROIs were selected by independent radiology technologists. Tumor/Normal $(\mathrm{T} / \mathrm{N})$ ratios were calculated as the ratios of radioactivity in the ROIs. The diagnostic abilities of the $\mathrm{T} / \mathrm{N}$ ratios for malignancy were analyzed by an ROC analysis using the ROCKIT1.1B2 Beta and PlotROC.xls software programs (University of Chicago). The area z-score $(\mathrm{Az})$ values were calculated from the areas under the ROC curves and the diagnostic accuracy was compared between each imaging modality. Any differences in the Az values were analyzed using the bivariate $\mathrm{x} 2$ test.

\subsection{Results}

Figure 1 shows the ROC curves. The vertical axis is the true positive fraction (TPF) that means sensitivity and the horizontal axis is the false positive fraction (FPF) that is same value of 1-specificity. The ROC curve of the early MIBI shifted to upper right and the sensitivity was better than the other in the high FPF area, however the sensitivity was low in the low FPF area. The ROC curve of the delayed MIBI shifted to the upper left and showed well balanced high sensitivity and specificity. The ROC curves of the early and delayed $\mathrm{Tl}$ were almost same and showed lower sensitivity and specificity than that of the delayed MIBI in most areas. 


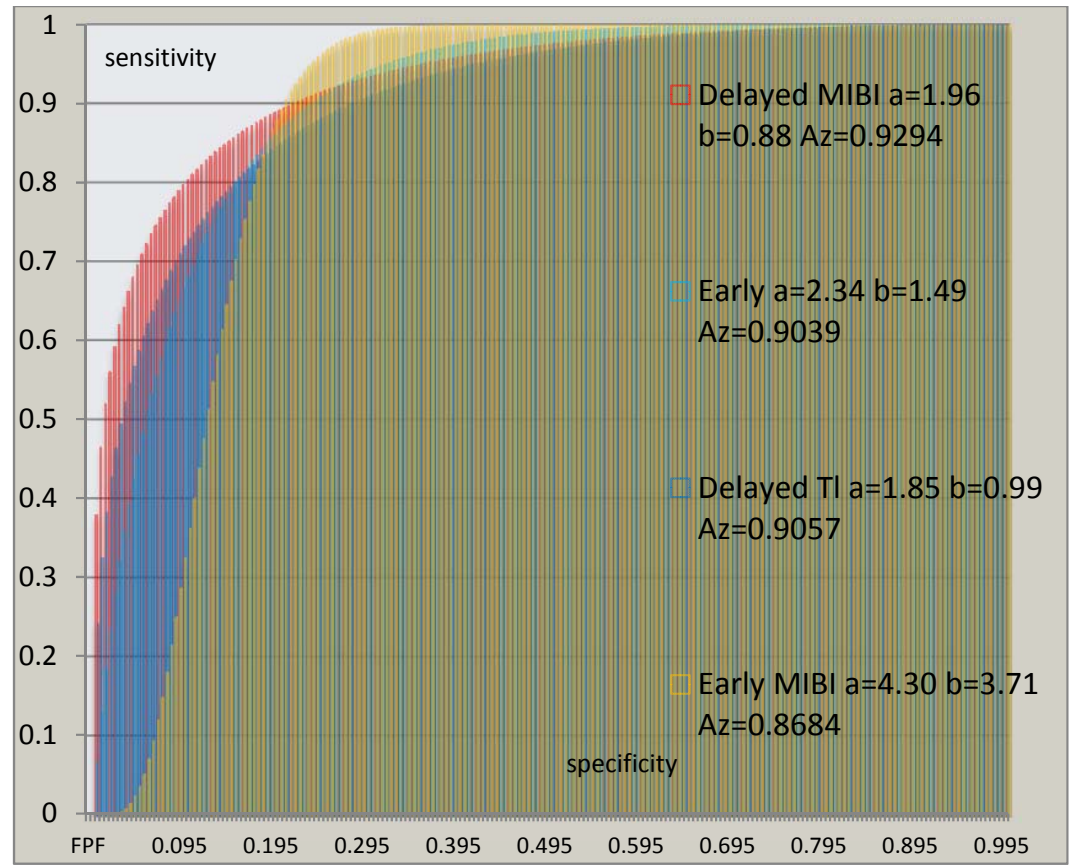

Figure 1. Receiver operating characteristic (ROC) curves

\subsection{Discussion}

\subsection{1. $\mathrm{Tl}$ SPECT}

T1 SPECT is useful for identifying the presence of a tumor [11], tumor malignancy $[1,6,12]$ and for making a differential diagnosis to distinguish tumor recurrence from radiation necrosis $[6,12,13]$. The $\mathrm{Tl}$ index, the ratio of radioactivity of ROI at the lesion and normal brain, was used to differentiate low and high grade glioma [1], recurrence and radiation necrosis [2, 3]. The dynamic Tl SPECT is reported to be useful to evaluate tumor vascularity, histology and malignancy $[14,15]$. However there are some false positive and false negative cases reported $[6,7,12,16,17]$. Inflammation after surgery or radiation is a major cause of false positive Tl uptake. The causes of false negative findings may include small tumor size, histological heterogeneity, cystic or necrotic components, or a low threshold of the detector or imaging [18, 19]. Both of central neurocytoma and ganglioglioma are benign glioma. High Tl uptake in a central neurocytoma and ganglioglioma were reported [20] [21]. High cell density and high metabolic rate are thought to explain the high Tl uptake in these low proliferative tumors. In the present series, one patient with a central neurocytoma showed high Tl uptake and no MIBI uptake. In this case, MIBI SPECT was more accurate than TI SPECT to evaluate tumor malignancy, because central neurocytoma is a benign tumor. Pilocytic astrocytoma is one of 
the most benign gliomas and the Tl SPECT findings of a pilocytic astrocytoma are reported to show a variable uptake [8].

\subsubsection{Tc-MIBI SPECT}

Tc-MIBI SPECT is reported to be useful to diagnose brain tumor recurrence [22, 23], high Sphase fraction and aneuploidy [24], tumor volume and survival [25, 26], and, differential diagnosis of radiation necrosis [27]. The Tc-MIBI index, the ratio of radioactivity of the ROI at the lesion and normal brain, was used to differentiate low and high grade gliomas [28], recurrence and radiation necrosis [22] and the estimation of the prognosis [25, 26]. However there are some false positive and false negative cases reported [23]. Tc-MIBI is concentrated in the mitochondria as the result of active diffusion due to increased metabolic needs [22]. TcMIBI uptake is determined by tumor malignancy, viability, density, oxygenation, vascular supply, and blood brain barrier (BBB) disruption [29]. These factors are not linearly correlated, because glioblastoma, the most malignant form of glioma, is pathologically heterogeneous including internal necrosis. False negative MIBI SPECT may occur due to the lack of contrast uptake on MRI and masked by other normal tissue uptake. This was seen in temporal and periventricular tumors [23], because Tc-MIBI is physiologically taken into the orbita, nasopharyngeal tissues, pituitary, scalp and choroid plexus [30]. Some of the false positive results were due to recent radiation induced local disruption of the BBB [23].

The pixel size of MIBI SPECT was smaller than that of Tl SPECT in this study. Therefore, MIBI SPECT has a higher spatial resolution than Tl SPECT. This study investigated the diagnostic ability of SPECT for glioma malignancy. Higher spatial resolution might influence the diagnostic ability with small or heterogeneous tumors. However, all of the gliomas in this study were larger than the pixel size. In addition, most of the malignant tumors in this study were highly heterogeneous in both of MRI and SPECT. Only the higher spatial resolution of MIBI SPECT could not explain the slightly higher diagnostic ability of MIBI SPECT than that of Tl SPECT. Some authors have reported that Tc-MIBI SPECT has higher sensitivity and specificity than that of Tl SPECT for adult and childhood brain tumors and differential diagnosis of recurrence and radiation necrosis [26, 27, 31]. However, other authors did not [32]. This discrepancy may be caused by small and heterogeneous patient populations and arbitrary selected cut off values. In Tl SPECT there is some normal brain uptake, this makes $\mathrm{T} / \mathrm{N}$ ratio low. TcMIBI has high photon energy level and higher tumor/background ratio in comparison with Tl SPECT and yields clear SPECT images and high sensitivity for malignant brain tumor $[22,27,31]$.

P-glycoprotein is one of the drug efflux pumps in the cell membrane and it acts to remove TcMIBI from tumor cells $[33,34]$. Other studies have suggested that p-glycoprotein expression in malignant glioma is the cause of false negative with Tc-MIBI SPECT [35-37]. The effect of pglycoprotein expression on clinical Tc-MIBI SPECT images has been investigated, and this effect was negligible in the diagnosis of brain tumor malignancy [38]. Henze also reported that p-glycoprotein efflux does not contribute to false negative MIBI SPECT, since MIBI washout did not occur between the early and late SPECT scans [39]. 


\subsubsection{ROC analysis}

Many facilities use a cut off value to evaluate tumor malignancy in Tl or Tc-MIBI SPECT [1, 4-8]. Serizawa reported that simple inter-institutional comparisons of $\mathrm{Tl}$ indices are not possible because measurement methods are different in each institute [7].

Most diagnostic tests have some errors and the results are influenced by arbitrary selected cut off values. ROC analysis is useful to evaluate the diagnostic values of each examination that yield some false positive or false negative results $[9,10]$. Henze reported that ROC analysis comparing I-123-iodo- $\alpha$-methyl-L-tyrosine (IMT), Tc-MIBI SPECT and F-18-fluorodeoxyglucose positron emission computed tomography (FDG PET) for detection of tumor progression in irradiated low-grade astrocytoma demonstrated that IMT yielded best diagnostic accuracy [29]. ROC analysis has better diagnostic power than the arbitrary cut off method and independent disease prevalence and decision-making threshold [29]. ROC analysis also provides adequate cut off value with appropriate sensitivity and specificity. Because SPECT image accuracy is dependent on SPECT machine quality, acquisition algorithm, and injected isotope dose, each facility should determine its own cut off value to provide an adequate diagnosis from an ROC analysis of own data.

Some facilities omitted the acquisition of delayed images due to their limited examination time. However, the current results demonstrated the superior diagnostic value of delayed images in both of Tl and Tc-MIBI SPECT. These results revealed both Tl and Tc-MIBI SPECT are useful for the diagnosis of gliomas. However, the SPECT study is not perfect, there are still some false positive and false negative findings. Multi-modality imaging studies may therefore help to diagnose brain tumors more correctly. If it is necessary to limit SPECT examinations due to medical economical issues related to the health insurance policy, then Tc-MIBI SPECT with a delayed acquisition is therefore considered to be the most reasonable choice.

\subsection{Conclusion}

Both of Tl and Tc-MIBI SPECT are useful imaging modalities for the evaluation of glioma malignancies. Although there was no statistically significant difference, delayed Tc-MIBI SPECT demonstrated the best diagnostic value in our patients with glioma based on an ROC analysis.

\section{Coregistration of functional SPECT images and high resolution anatomical MRI}

\subsection{Introduction}

201Thallium Single Photon Emission Computed Tomography (Tl SPECT) is a useful functional imaging modality for the diagnosis of malignancy and activity of brain tumors. One of the drawbacks of Tl SPECT is its low spatial resolution. The coregistration of functional SPECT images and high resolution anatomical magnetic resonance imaging (MRI) is considered to be a reasonable method to improve the low spatial resolution of SPECT. 
Some methods developed for the coregistration of multiple images have been reported. One is a prospective method using external markers[40-42]. Another is the retrospective methods based on imaging data[43-46]. Prospective methods need prospective external fiducial marker placement and only the image with the marker can be coregistered. Retrospective methods do not need external markers, and therefore they are more flexible. Variable images can be retrospectively coregistered using software programs.

A newly developed software Brain Easy Analysis Tool for 201Tl-SPECT (BEAT-Tl, Fuji Film RI Pharma. Tokyo, Japan) was used for the coregistration of Tl SPECT and MRI in patients with brain tumors. The feasibility and reliability of BEAT-Tl was evaluated for patients with brain tumors.

\subsection{Materials and methods}

The patient population included 98 patients ( 47 men and 51 women). The age range was from 4 to 84 years of age and the median age was 54 . All patients had been admitted to Tsukuba University Hospital between 2004 and 2007. The brain MRI and Tl SPECT images were taken within a 1 week interval. No anti-tumor therapy was administered between the examinations. The sets of MRI and TI SPECT examinations were taken for 120 times (59 times for men and 61 times for women). MRI showed mass lesions in all of the patients. The final diagnoses included glioma, lymphoma, neurocytoma, metastasis, meningioma, radiation necrosis, encephalitis and multiple sclerosis. Most patients underwent tumor removal surgery and the pathological diagnoses were determined.

MRI was performed using the 1.5 Tesla clinical MRI scanner (Gyroscan NT Intera, Philips, Netherlands). T1 weighted images with or without Gd-DTPA enhancement, T2 weighted images and Fluid attenuated inversion recovery images (FLAIR) were routinely acquired. Tl SPECT images were acquired 15 minutes (early image) and 3 hours (delayed image) after the intravenous injection of $74 \mathrm{MBq}$ of Thallium Chloride (Fuji Film RI Pharma. Tokyo, Japan) using a multi-detector SPECT machine (E.CAM, Siemens Medical, Erlangen, Germany) and a high resolution collimator (LEHR, Siemens Medical, Erlangen, Germany). The Butterworth pre-correction filter and the Chang method were used for pre and post attenuation corrections. The Ramp filter was used for reconstruction. The image matrixes for Tl SPECT were $64 \times 64$. Both the pixel sizes and the slice thickness for Tl SPECT were $6.61 \mathrm{~mm}$.

The MRI and SPECT data were saved as Digital Imaging and Communications in Medicine (DICOM) formats. The DICOM data were converted to the analyze format using File Converter Ver. 2.5 (Fuji Film RI Pharma. Tokyo, Japan). Both the MRI and SPECT data in analyze format were transferred to the co-registration software program BEAT$\mathrm{Tl}$ and then coregistered images were created. The BEAT-Tl program is based on Statistical Parametric Mapping (SPM) and it can be used on personal computers with the Windows operating system. The imaging quality, clinical usefulness and the artifacts of the coregistered images were evaluated by visual inspection. 


\subsection{Results}

The coregistered images were easily and promptly created within one minute. The image quality was sufficient. There were no large displacements of the MRI and SPECT images and no major artifacts. The coregistration was fully automatic and no manual coregistration procedure was required.

The small hot uptake in Tl SPECT always coincided with enhanced tumors in MRI. For large tumors, Gd enhanced T1 weighted MRI showed relatively homogeneous enhancement. However, Tl SPECT showed focal uptake of $\mathrm{Tl}$ in the homogeneously enhanced tumor. Therefore, the coregistered images demonstrated metabolic heterogeneity on Tl SPECT. In most patients, $\mathrm{Tl}$ hot uptake was in the Gd enhanced tumor. In some patients, Tl uptake was observed outside of the Gd enhanced tumor.

\subsection{Illustrative cases}

\section{Case 1:A 64-year-old female with an anaplastic oligodendroglioma}

Tl uptakes were different between 2 fusion images of Gd enhanced T1 weighted MRI and early Tl SPECT (Fig. 2, Lt) or delayed Tl SPECT (Fig.2,Rt). Tl uptakes were heterogeneous even though the Gd enhanced tumor was homogenous. Most $\mathrm{Tl}$ uptakes were within the Gd enhanced tumor. However the part of the delayed Tl uptake was out of the Gd enhanced area, and therefore this region seemed to demonstrate tumor invasion into normal brain tissue.
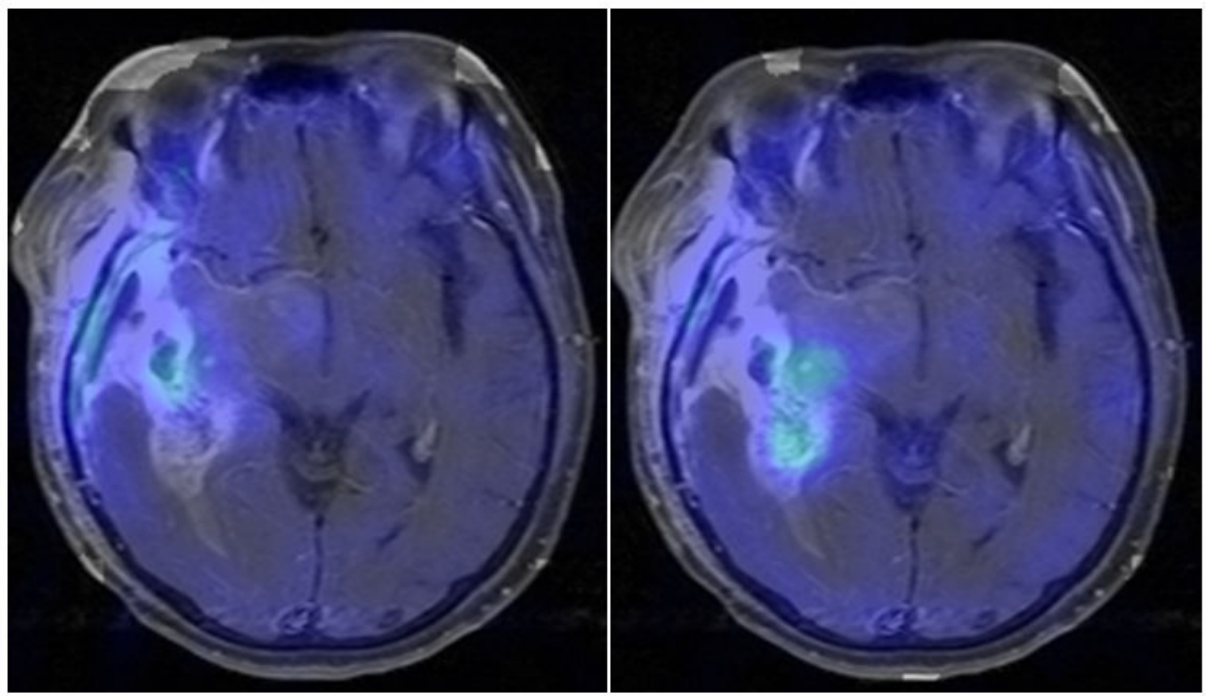

Figure 2. Fusion images of Gd enhanced T1 weighted MRI and early TI SPECT (Lt) or delayed TI SPECT (Rt) of Case 1. 


\section{Case 2: A 34-year-old male with a frontal glioblastoma.}

A fusion image of the preoperative Gd enhanced MRI and delayed Tl SPECT (Fig 3, Lt) showed Rt medial Tl uptake although the Gd enhanced tumor was located in the corpus callosum. Because there was a tumor without Gd enhancement in the left frontal lobe, the left frontal lobe and the tumor at the corpus callosum was removed. After radiation therapy of $60 \mathrm{~Gy}, 2$ months after the surgery, tumor recurrence was seen mainly in the Rt medial frontal lobe where the hot Tl uptake was seen in Gd enhanced T1 weighted MRI (Fig 3, Rt).
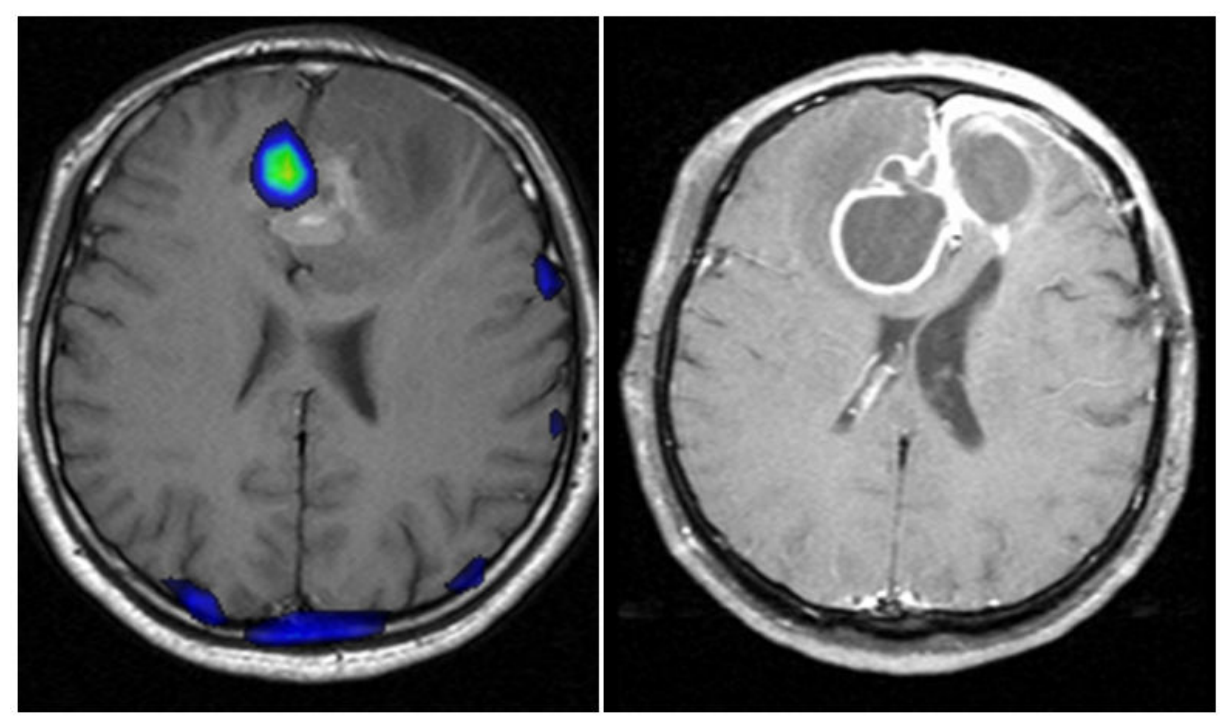

Figure 3. Fusion image of preoperative Gd enhanced MRI and delayed TI SPECT (Lt) and Gd enhanced T1 weighted MRI (Rt) 2 months after the surgery.

\subsection{Discussion}

\subsubsection{Usefulness of coregistration}

The one of the major disadvantages of SPECT is the low spatial resolution caused by scattering radiation. Because no anatomical landmarks are seen in Tl SPECT images, it is sometimes difficult to precisely determine the locations of lesions and the relationships with normal structures based on only Tl SPECT images. The coregistration of Tl SPECT and MRI has thus completely solved these problems.

Even when tumors were found to be homogeneous in MRI, Tl SPECT showed metabolic heterogeneity. These detailed analyses thus become possible by studying coregistration images. Further pathological and clinical studies are expected to reveal the clinical significance of these imaging modalities. 


\subsubsection{Method of coregistration}

Other methods for the coregistration of SPECT and MRI has been reported [45-47]. These methods required meticulous and cumbersome procedures and long calculation time. In some cases sufficient coregistration could not be achieved by automatic calculations and thus required manual procedures.

The Brain easy analysis tool (BEAT, Fuji film RI Pharma, Tokyo, Japan) is the coregistration program for cerebral blood flow (CBF) SPECT and MRI. BEAT uses automated image registration (AIR http://bishopw.loni.ucla.edu/) as the coregistration algorithm and ratio image uniformity (RIU) as the cost function. In CBF SPECT image, tracer uptake into normal brain tissue is seen, so the contour of the brain tissue is clear. However, in the brain tumor SPECT image, the tracer uptake into the normal brain tissue is negligible, so the contour of the normal brain tissue is not seen. Because of these factors, the coregistration of brain tumor SPECT and MRI has not been achieved using the same algorithm used for the coregistration of CBF SPECT image and MRI.

BEAT-Tl uses statistical parametric mapping (SPM) 2 (http://www.fil.ion.ucl.ac.uk/spm/) as the voxel based coregistration software and normalized mutual information (NMI) as the cost function to evaluate coregistration accuracy. These algorithms allow automatic coregistration of brain tumor SPECT and MRI with satisfactory accuracy.

The BEAT-Tl program runs on a normal personal computer without any problems. The usual calculation time is within a minute, so it is simple for operators. The results could be fed back to a busy daily clinical practice, so the clinical impact and efficacy are significant. In the current study, the accuracy of the coregistration was satisfactory. There were no major errors or artifacts.

SPECT/computed tomography (CT) or positron emission CT (PET)/CT are thus considered to be alternative methods to create coregistration images. These hardware based coregistrations must be more accurate than the software based coregistrations. Currently, however, these machines are expensive and not available in most clinical settings. As the results, the coregistration software program is both a convenient and sufficiently accurate method to create coregistration images in most clinical facilities.

\subsubsection{Study limitation}

The current study population was relatively small and this study did not evaluate every patient admitted to this hospital. In the future, a prospective study will reveal the exact usefulness and the limitations of the coregistration images of TI SPECT and MRI.

The uptake of $\mathrm{Tl}$ into a lesion is affected by multiple factors, including tumor malignancy, tumor metabolism, cell density, blood brain barrier breakdown, blood flow, blood volume and capillary density [1,5]. Therefore, the interpretation of Tl SPECT should be done cautiously. The fusion of Tl SPECT and MRI could be helpful to evaluate the mechanisms of $\mathrm{Tl}$ uptake into lesions. Although no large displacements of the coregistration were seen in the images created by BEAT-Tl, the coregistration error was not 
measured in this study. In order to investigate the coregistration error, stereotactic or image guided surgery will be done in a future study. One of the problems of image study for malignant brain tumors is the heterogeneity. Glioblastoma multiforme is pathologically, metabolically, and radiologically heterogeneous. Pathological radiological correlations could reveal the meaning of each finding. Neurosurgeons could remove the tumor; however, the registration of the removed tissue at exact locations on each image is not easy. Image guided surgery using coregistration image should facilitate these studies. The application of fusion images for navigation guided neurosurgery will be developed.

\subsection{Conclusion}

BEAT-Tl is a useful coregistration software program that is used to evaluate brain tumors. It improves the spatial correlation of SPECT images and such coregistration images demonstrate a metabolic heterogeneity in tumors that is not revealed in MRI.

\section{Evaluation of glioma surgical extent using fusion image of T1 SPECT and anatomical MRI}

\subsection{Introduction}

In glioma surgery, removal extent has been evaluated using morphological image, such as MRI or X-ray CT. These morphological images could not completely evaluated surgical removal extent, because glioma is invasive tumor. The contribution of glioma surgery for the improvement of the prognosis is controversial $[48,49]$. One of the causes of this discrepancy may be the method of evaluation of glioma surgical extent. We evaluated the glioma surgical extent using fusion image of metabolic Tl SPECT and anatomical MRI. Our hypothesis is these fusion images could more precisely predict the prognosis of the patient received glioma removal surgery.

\subsection{Methods}

From 2006 to 2008, 146 patients examined both of Tl SPECT and MRI at the same time under the diagnosis of brain tumor. And 58 patients received tumor removal surgery after the examinations. Only 36 patients who were examined MRI within 3 days after the surgery were included because surgical modification of MRI increase after postoperative 4 days [50]. Finally 30 patients who have Gd-DTPA enhanced tumor in MRI and hot tumor uptake in TI SPECT were included in this study, in order to make image evaluation easy. Final patient population included 17 male and 13 female, age range from 34 to 80 year-old. World Health Organization (WHO) pathological grade are 10 grade 3 and 20 grade 4 . Oligodendroglioma component was recognized in 7 grade 3 tumors. All patients received adjuvant standard radiation and chemotherapy. 


\subsection{Results}

Partial removal group were frequently seen in Grade 4 . The removal rates with MRI and SPECT were concordant for 24 cases. The removal rates with MRI were higher than those with SPECT in 6 cases. So SPECT more efficiently revealed residual tumor than MRI did (Figure 4,5). The time to progression was short in partial removal group with MRI and SPECT (Figure 6). The removal rates, pathology and oligodendroglioma components were all related with the prognosis in multivariate analysis.

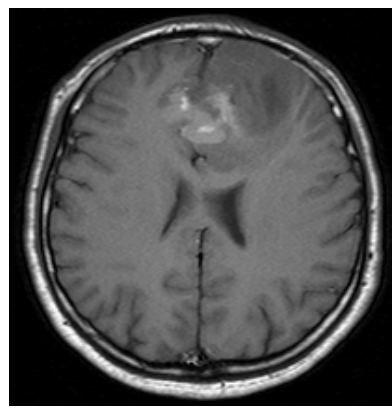

(a)

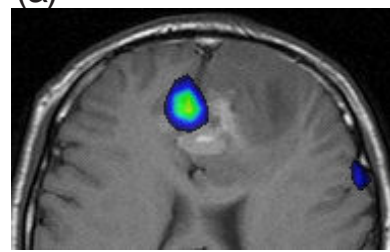

(b)

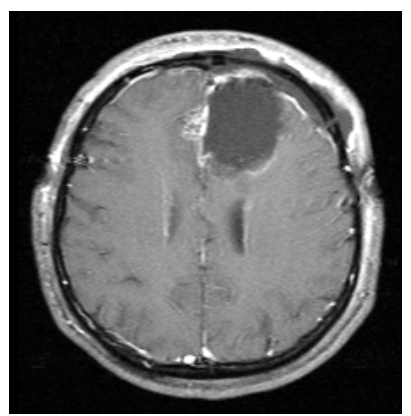

(c)

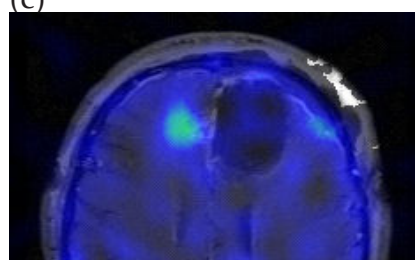

(d)

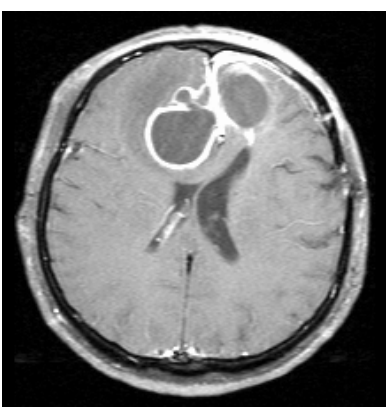

(e)

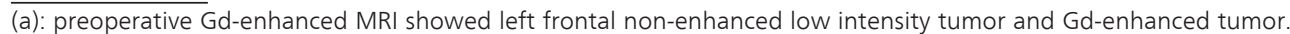
Enhanced tumor located at left medial frontal lobe and invaded into right medial frontal lobe.

(b): The fusion image of preoperative TI-SPECT and MRI showed hot TI uptake only at right medial frontal lobe.

(c): Postoperative Gd-enhanced MRI showed small residual enhanced tumor at right medial frontal lobe.

(d): The fusion image with TI-SPECT demonstrated residual tumor at right medial frontal lobe. Surgical extents were evaluated as subtotal removal by MRI, partial removal with TI-SPECT.

(e): After radiation and chemotherapy, tumor recurrence occurred from right medial frontal residual tumor.

(Reproduced from Y Shibata, CI research 32:19-24, 2010 with permission.)

Figure 4. Case 1, 34 year-old man with glioblastoma. (same case as Fig 2-2) 


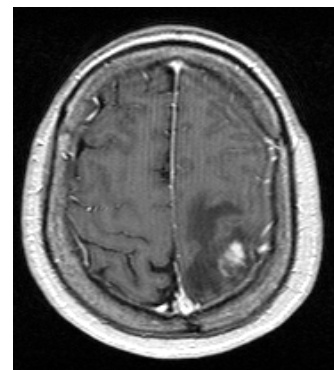

(a)

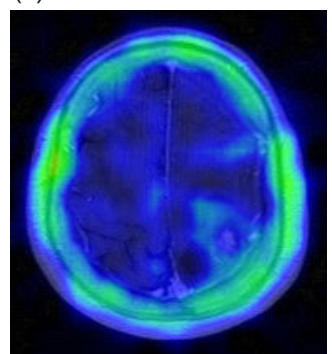

(b)

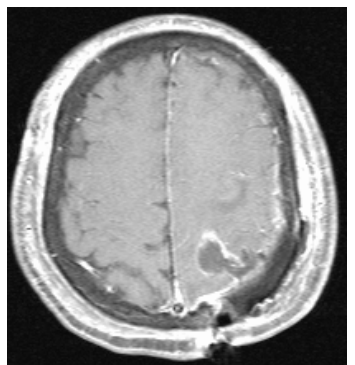

(c)

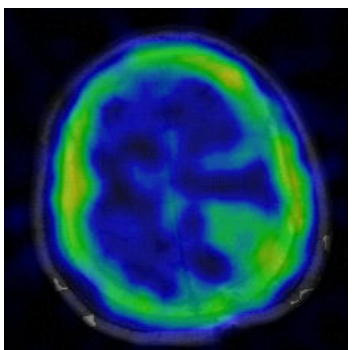

(d)

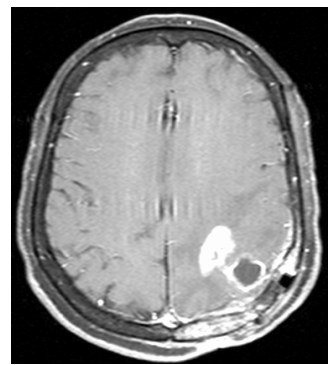

(e)

(a): Preoperative Gd-enhanced MRI showed right parietal ring enhanced tumor and perifocal edema.

(b): The fusion image of preoperative Gd-enhanced MRI and TI SPECT

(c): Postoperative Gd-enhanced MRI showed small residual enhanced tumor at right parietal lobe.

(d): The fusion image of preoperative TI SPECT and MRI showed hot TI uptake at right residual parietal lobe.

(e): After radiation and chemotherapy, tumor recurrence occurred from hot Tl uptake area.

(Reproduced from Y Shibata, Cl research 32:19-24, 2010 with permission.)

Figure 5. Case 2, 57 year-old woman with glioblastoma.
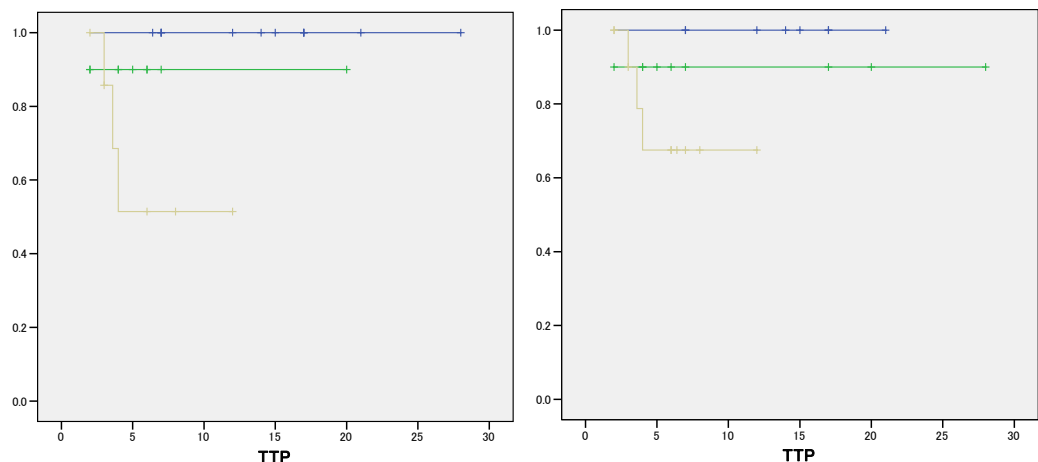

Figure 6. Kaplan-Meier curves of Time to Progression evaluated by removal rate in MRI (Left) and SPECT (Right). Partial removal groups (brown curves) showed significantly early recurrences than both of total (blue curves) and sub-total removal groups (blue curves). 


\subsection{Conclusion}

The fusion image of preoperative Tl SPECT and postoperative MRI is more useful to evaluate glioma removal extent than MRI only. Partial removal groups have poor prognosis, so maximum surgical removal should be aimed using multimodal images including MRI and Tl SPECT. The total removal of T1 SPECT positive lesion improves the prognosis and especially prevents early recurrence.

\section{Acknowledgements}

I appreciate my mentors, collaborators and the patients.

\section{Author details}

Yasushi Shibata

Department of Neurosurgery, Mito Medical Center, University of Tsukuba, Ibaraki, Japan

I declare that I have no conflict of interest.

\section{References}

[1] Black KL, Hawkins RA, Kim KT, Becker DP, Lerner C, Marciano D. Use of thallium-201 SPECT to quantitate malignancy grade of gliomas. J Neurosurg. 1989;71:342-6.

[2] Kosuda S, Shioyama Y, Kamata N, Suzuki K, Tanaka Y, Nakamura O, et al. [Differential diagnosis between recurrence of brain tumor and radiation necrosis by 201Tl SPECT]. Nippon Igaku Hoshasen Gakkai Zasshi. 1991;51:415-21. (In Japaese)

[3] Kosuda S, Fujii H, Aoki S, Suzuki K, Tanaka Y, Nakamura O, et al. Reassessment of quantitative thallium-201 brain SPECT for miscellaneous brain tumors. Ann Nucl Med. 1993;7:257-63.

[4] Kahn D, Follett KA, Bushnell DL, Nathan MA, Piper JG, Madsen M, et al. Diagnosis of recurrent brain tumor: value of $201 \mathrm{Tl}$ SPECT vs 18F- fluorodeoxyglucose PET. Am J Roentgenol. 1994;163:1459-65.

[5] Burkard R, Kaiser KP, Wieler H, Klawki P, Linkamp A, Mittelbach L, et al. Contribution of thallium-201-SPECT to the grading of tumorous alterations of the brain. Neurosurg Rev. 1992;15:265-73. 
[6] Staffen W, Hondl N, Trinka E, Iglseder B, Unterrainer J, Ladurner G. Clinical relevance of 201Tl-chloride SPET in the differential diagnosis of brain tumours. Nucl Med Commun. 1998;19:335-40.

[7] Serizawa T, Saeki N, Higuchi Y, Ono J, Matsuda S, Sato M, et al. Diagnostic value of thallium-201 chloride single-photon emission computerized tomography in differentiating tumor recurrence from radiation injury after gamma knife surgery for metastatic brain tumors. J Neurosurg. 2005;102 Suppl:266-71.

[8] Comte F, Bauchet L, Rigau V, Hauet JR, Fabbro M, Coubes P, et al. Correlation of preoperative thallium SPECT with histological grading and overall survival in adult gliomas. Nucl Med Commun. 2006;27:137-42.

[9] Metz CE. ROC methodology in radiologic imaging. Invest Radiol. 1986;21:720-33.

[10] Metz CE, A. HB, Jong-Her S. Maximum likelihood estimation of receiver operating characteristic (ROC) curves from continuously-distributed data. Statistics in Medicine. 1998;17:1033-53.

[11] O'Tuama LA, Janicek MJ, Barnes PD, Scott RM, Black PM, Sallan SE, et al. 201Tl/ 99mTc-HMPAO SPECT imaging of treated childhood brain tumors. Pediatr Neurol. 1991;7:249-57.

[12] Yoshii Y, Satou M, Yamamoto T, Yamada Y, Hyodo A, Nose T, et al. The role of thallium-201 single photon emission tomography in the investigation and characterisation of brain tumours in man and their response to treatment. Eur J Nucl Med. 1993;20:39-45.

[13] Schwartz RB, Carvalho PA, Alexander E, 3rd, Loeffler JS, Folkerth R, Holman BL. Radiation necrosis vs high-grade recurrent glioma: differentiation by using dual-isotope SPECT with $201 \mathrm{TI}$ and 99mTc-HMPAO. AJNR Am J Neuroradiol. 1991;12:1187-92.

[14] Ueda T, Kaji Y, Wakisaka S, Watanabe K, Hoshi H, Jinnouchi S, et al. Time sequential single photon emission computed tomography studies in brain tumour using thallium-201. Eur J Nucl Med. 1993;20:138-45.

[15] Sugo N, Yokota K, Kondo K, Harada N, Aoki Y, Miyazaki C, et al. Early dynamic 201Tl SPECT in the evaluation of brain tumours. Nucl Med Commun. 2006;27:143-9.

[16] Buchpiguel CA, Alavi JB, Alavi A, Kenyon LC. PET versus SPECT in distinguishing radiation necrosis from tumor recurrence in the brain. J Nucl Med. 1995;36:159-64.

[17] Rollins NK, Lowry PA, Shapiro KN. Comparison of gadolinium-enhanced MR and thallium-201 single photon emission computed tomography in pediatric brain tumors. Pediatr Neurosurg. 1995;22:8-14. 
[18] Kallen K, Burtscher IM, Holtas S, Ryding E, Rosen I. 201Thallium SPECT and 1HMRS compared with MRI in chemotherapy monitoring of high-grade malignant astrocytomas. J Neurooncol. 2000;46:173-85.

[19] Young RJ, Ghesani MV, Kagetsu NJ, Derogatis AJ. Lesion size determines accuracy of thallium-201 brain single-photon emission tomography in differentiating between intracranial malignancy and infection in AIDS patients. AJNR Am J Neuroradiol. 2005;26:1973-9.

[20] Kumabe T, Shimizu H, Sonoda Y, Shirane R. Thallium-201 single-photon emission computed tomographic and proton magnetic resonance spectroscopic characteristics of intracranial ganglioglioma: three technical case reports. Neurosurgery. 1999;45:183-7.

[21] Kanamori M, Kumabe T, Shimizu H, Yoshimoto T. (201)Tl-SPECT, (1)H-MRS, and MIB-1 labeling index of central neurocytomas: three case reports. Acta Neurochir (Wien). 2002;144:157-63.

[22] Soler C, Beauchesne P, Maatougui K, Schmitt T, Barral FG, Michel D, et al. Technetium-99m sestamibi brain single-photon emission tomography for detection of recurrent gliomas after radiation therapy. Eur J Nucl Med. 1998;25:1649-57.

[23] Le Jeune FP, Dubois F, Blond S, Steinling M. Sestamibi technetium-99m brain singlephoton emission computed tomography to identify recurrent glioma in adults: 201 studies. J Neurooncol. 2006;77:177-83.

[24] Ak I, Gulbas Z, Altinel F, Vardareli E. Tc-99m MIBI uptake and its relation to the proliferative potential of brain tumors. Clin Nucl Med. 2003;28:29-33.

[25] Beauchesne P, Soler C. Correlation of 99mTc-MIBI brain spect (functional index ratios) and survival after treatment failure in malignant glioma patients. Anticancer Res. 2002;22:3081-5.

[26] Beauchesne P, Pedeux R, Boniol M, Soler C. 99mTc-sestamibi brain SPECT after chemoradiotherapy is prognostic of survival in patients with high-grade glioma. J Nucl Med. 2004;45:409-13.

[27] Yamamoto Y, Nishiyama Y, Toyama Y, Kunishio K, Satoh K, Ohkawa M. 99mTc-MI$\mathrm{BI}$ and 201TI SPET in the detection of recurrent brain tumours after radiation therapy. Nucl Med Commun. 2002;23:1183-90.

[28] Baillet G, Albuquerque L, Chen Q, Poisson M, Delattre JY. Evaluation of single-photon emission tomography imaging of supratentorial brain gliomas with technetium-99m sestamibi. Eur J Nucl Med. 1994;21:1061-6.

[29] Henze M, Mohammed A, Schlemmer HP, Herfarth KK, Hoffner S, Haufe S, et al. PET and SPECT for Detection of Tumor Progression in Irradiated Low-Grade Astrocytoma: A Receiver-Operating-Characteristic Analysis. J Nucl Med. 2004;45:579-86. 
[30] Kirton A, Kloiber R, Rigel J, Wolff J. Evaluation of pediatric CNS malignancies with (99m)Tc-methoxyisobutylisonitrile SPECT. J Nucl Med. 2002;43:1438-43.

[31] O'Tuama LA, Treves ST, Larar JN, Packard AB, Kwan AJ, Barnes PD, et al. Thallium-201 versus technetium-99m-MIBI SPECT in evaluation of childhood brain tumors: a within-subject comparison. J Nucl Med. 1993;34:1045-51.

[32] Nishiyama Y, Yamamoto Y, Fukunaga K, Satoh K, Kunishio K, Ohkawa M. Comparison of 99Tcm-MIBI with $201 \mathrm{Tl}$ chloride SPET in patients with malignant brain tumours. Nucl Med Commun. 2001;22:631-9.

[33] Feun LG, Savaraj N, Landy HJ. Drug resistance in brain tumors. J Neurooncol. 1994;20(2):165-76.

[34] Lehnert M. Multidrug resistance in human cancer. J Neurooncol. 1994;22(3):239-43.

[35] Andrews DW, Das R, Kim S, Zhang J, Curtis M. Technetium-MIBI as a glioma imaging agent for the assessment of multi-drug resistance. Neurosurgery. 1997;40(6): 1323-32.

[36] Ballinger JR, Sheldon KM, Boxen I, Erlichman C, Ling V. Differences between accumulation of 99mTc-MIBI and 201Tl-thallous chloride in tumour cells: role of P-glycoprotein. Q J Nucl Med. 1995;39(2):122-8.

[37] Piwnica-Worms D, Chiu ML, Budding M, Kronauge JF, Kramer RA, Croop JM. Functional imaging of multidrug-resistant P-glycoprotein with an organotechnetium complex. Cancer Res. 1993;53(5):977-84.

[38] Shibata Y, Matsumura A, Nose T. Effect of expression of P-glycoprotein on technetium-99m methoxyisobutylisonitrile single photon emission computed tomography of brain tumors. Neurol Med Chir (Tokyo). 2002;42:325-30.

[39] Henze M, Mohammed A, Schlemmer H, Herfarth KK, Mier W, Eisenhut M, et al. Detection of tumour progression in the follow-up of irradiated low-grade astrocytomas: comparison of 3-(123I)iodo-alpha-methyl- L-tyrosine and 99mTc-MIBI SPET. Eur J Nucl Med Mol Imaging. 2002;29:1455-61.

[40] Yamamoto Y, Nishiyama Y, Monden T, Matsumura Y, Satoh K, Ohkawa M. Clinical Usefulness of Fusion of 131I SPECT and CT Images in Patients with Differentiated Thyroid Carcinoma. J Nucl Med. 2003;44:1905-10.

[41] Chajari M, Lacroix J, Peny AM, Chesnay E, Batalla A, Henry-Amar M, et al. Gallium-67 scintigraphy in lymphoma: is there a benefit of image fusion with computed tomography? Eur J Nucl Med Mol Imaging. 2002;29:380-7.

[42] Aqueveque AC, Gonzalez EP, Gutierrez BD, Jaimovich FR, Diaz PJ, Csendes GP, et al. (Fusion of SPECT with computed tomography or magnetic resonance for the interpretation of abnormal tracer uptake.). Rev Med Chil. 2007 (In Spanish);135:725-34. 
[43] Pietrzyk U, Herholz K, Fink G, Jacobs A, Mielke R, Slansky I, et al. An interactive technique for three-dimensional image registration: validation for PET, SPECT, MRI and CT brain studies. J Nucl Med. 1994;35:2011-8.

[44] Pietrzyk U, Herholz K, Schuster A, Stockhausen H-Mv, Lucht H, Heiss W-D. Clinical applications of registration and fusion of multimodality brain images from PET, SPECT, CT, and MRI. European Journal of Radiology. 1996;21:174-82.

[45] Sabbah P, Foehrenbach H, Dutertre G, Nioche C, DeDreuille O, Bellegou N, et al. Multimodal anatomic, functional, and metabolic brain imaging for tumor resection. Clin Imaging. 2002;26:6-12.

[46] Komori T, Kanamoto T, Ogura Y, Utsunomiya K, Adachi I, Narabayashi I. The evaluation of thallium-201 SPECT/MRI image fusion in brain tumor. Rinsho Hoshasen. 2004;49:285-90. (In Japanese)

[47] Holman BL, Zimmerman RE, Johnson KA, Carvalho PA, Schwartz RB, Loeffler JS, et al. Computer-assisted superimposition of magnetic resonance and high-resolution technetium-99m-HMPAO and thallium-201 SPECT images of the brain. J Nucl Med. 1991;32:1478-84.

[48] Mitchell P, Ellison DW, Mendelow AD. Surgery for malignant gliomas: mechanistic reasoning and slippery statistics. Lancet Neurol. 2005;4:413-22.

[49] Sanai N, Berger MS. Glioma extent of resection and its impact on patient outcome. Neurosurgery. 2008;62:753-64; discussion 264-6.

[50] Albert FK, Forsting M, Sartor K, Adams HP, Kunze S. Early postoperative magnetic resonance imaging after resection of malignant glioma: objective evaluation of residual tumor and its influence on regrowth and prognosis. Neurosurgery. 1994;34:45-60; discussion -1. 
